\title{
A Comprehensive Approach for Multi Biometric Recognition Using Sclera Vein and Finger Vein
}

\author{
Saranya.K.R ${ }^{1}$, Vanitha. $S^{2}$, Selva priya.G ${ }^{3}$, Minojini.N ${ }^{4}$, Nivi.A.N $^{5}$ \\ Pg Scholar, Department of Computer Science and Engineering, Dr.NGP IT, Coimbatore, India ${ }^{1,3,4,5}$ \\ Assistant Professor, Department of Computer Science and Engineering, Dr.NGP IT, Coimbatore, India ${ }^{2}$
}

\begin{abstract}
Multimodal biometrics use more than one means of biometric identification to achieve higher detection accuracy, since sometimes a single biometric is not good enough used to do identification and codification. Sclera and finger print strain is a new counterpart vein recognition method using a two-stage parallel approach for documentation and bouting. First, modeled a rotation- and rule-invariant $\mathrm{Y}$ shape descriptor based feature extraction method to efficiently eliminate most unlikely matches. Second, developed a weighted polar line sclera descriptor structure to incorporate mask information to reduce Graphics processing unit (GPU) awareness cost. Third, modeled a coarse-tofine two-rung matching approach. Finally, developed a weighing scheme to map the subtasks to GPU processing things. The experimental upshots show that the urged method can achieve dramatic processing speed improvement without compromising the detection accuracy. Here examined the previously proposed finger-strain identification approaches and develop a new approach that illustrates it superiority over prior published efforts. Then is to progress and examine two new score-level blends, i.e., holistic and non linear fusion, and comparatively evaluate them with more popular score-level fusion approaches to ascertain their effectiveness in the proposed scheme. The aim is to combine the upshots obtained by different biometric traits and significantly improve the overall accuracy of the biometric system.
\end{abstract}

Keywords: Holistic, Fusion, Traits, GPU.

\section{INTRODUCTION}

Biometrics is a class of Pattern detection problem. biometrics that make it well-suited for non-compliant Biometrics is computerized method of identifying a person recognition situations [4][5] .A lot of techniques have been or verifying the identity of a person based on a applied for different applications [7]. description and physiological or behavioral characteristic [3]. strain verification becomes more and more important task for pattern is the network of blood vessels beneath a person's these applications especially in security systems.

pelt. This strain pattern can be used to authenticate the identity of an individual. In the ubiquitous artery civilization, where individuals can easily deal with their information anytime and anywhere, people are also faced with the peril that others can easily access the same information anytime and anywhere. Because of this risk, personal verification technology, which can distinguish between registered legitimate users and imposters, is now generating interest. Currently, countersigns, Personal Identification cards are used for personal verification. However, cards can be stolen, and countersigns and numbers can be guessed or forgotten. To solve these problems, biometric admittance computerization, which identifies people by their unique biological information, is attracting attention [6].

The importance of utilizing biometrics to establish personal authenticity and to detect impostors is growing in the present scenario of global refuge anxiety. Development of a biometric scheme for personal description, which fulfils the requirements for access control of secured areas and other applications like identity validation for social welfare, crime detection, ATM access, computer refuge, etc.. Sclera detection is identification of a human using the sclera, the "white of the eye.' It offers several benefits over other eye-based

Finger vein recognition is a method of biometric authentication that uses pattern-recognition techniques based on images of human finger vein patterns beneath the pelt's surface. Finger strain recognition is one of many forms of biometrics used to identify individuals and verify their identity [8]. Sclera vein recognition is more accurate than any other biometric and it improves the overall accuracy of the system without compromising recognition and it provides more security than any other biometrics [9]. The combination of both Sclera Vein and Finger Vein produce accurate results.

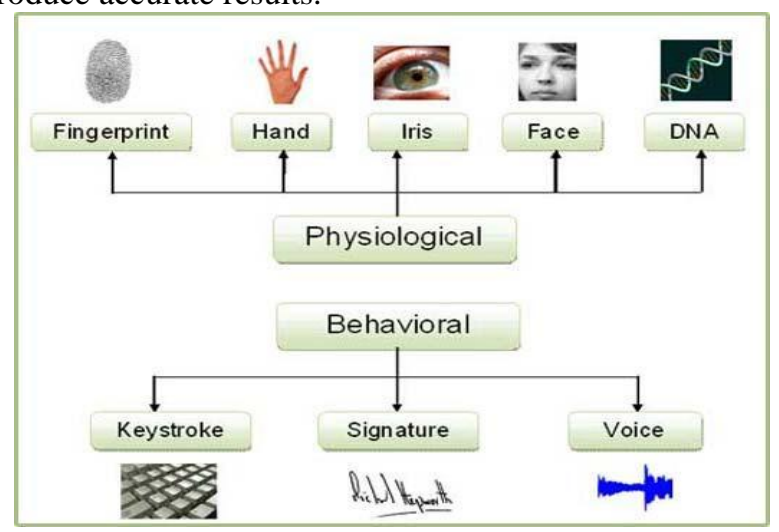

Fig.1.Biometrics Characteristic 


\section{RELATED WORK}

In previous works, identification of users using the sclera region has been referred to as 'conjunctival vasculature identification.' However, as the conjunctiva is the toprecord transparent layer of the sclera and images of the sclera region capture more than just this top-most layer, it is more specific to refer to the scheme as performing 'sclera identification'. In [10], Derakhshani et. al. first proposed using 'conjunctival vascular' patterns for user verification..

They used contrast limited adaptive histogram equalization (CLAHE) to enhance the green color plane of the RGB representation, and a multi-scale region-growing method to identify the sclera veins from the image circumstances. For matching, they proposed a multi-level matching approach a coarse approach to sub divide the database, and a fine approach to specifically match a user from the preliminary sub-divided segment of the database, as found from the course approach [11].

Later, in [12], Derakhshani and Ross used a texture-based neural grid classifier, manual separation of the sclera province, and adaptive thresholding and augmentation on the vasculature images. Most recently, in [13] Crihalmeanu et. al. used a semi-automated k-means clustering algorithm to estimate the sclera region from the RGB values of the pixels in the color sclera demonstrations, and used manual interference to correct for misclassified boundaries (in particular, the lower eyelid boundary). They projected a registration method that incorporates local affine and global smoothing transformations that locally deforms the template image to provide the best registration with the target image.

Finger Vein ID is a biometric authentication system that matches the vascular pattern in an individual's finger to previously achieved data. Hitachi matured and patented a finger vein ID system in 2005 [14]. David et al [15] imported exploratory process to enhance the image quality that worsen by light effect and produces noise by the web camera, then disjointed the strain template by using adaptive threshold method and matched them using improved template matching. Wenming et al [16] proposed a structured personal identification approach using finger vein Location and Direction Coding (LDC). Initially finger vein imaging device is designed using Near-InfraRed (NIR) light source, by which a database for finger strain representations is established. Hua-Bin et al [3] presented an algorithm based on adaptive filtering and retinex method for enhancement of hand vein images.

\section{PROPOSED WORK AND SIMULATION RESULTS}

It is an efficient approach based on multimodal biometrics (Sclera vein and fingerprint vein) for detection. The proposed scheme deals with two methods of extracting feature of veins properties from fingerprint vein and Sclera vein and gives the optimal solution. In particular, propose a protocol which integrates multi-biometrics, in which statistics from multiple biometric sources is combined. The proposed work uses multimodal biometrics for continuous authentication namely sclera blood vein pattern and fingerprint. For initial log in uses sclera blood veins as it is unique to each individual and it is more accurate than any other biometric. The experimental upshots show that sclera recognition is a promising new biometrics for positive human ID [18]. A new method for sclera segmentation which works for both color and grayscale images is proposed and, designed a Gabor wavelet-based sclera pattern enhancement method to emphasize and binarize the sclera vessel patterns.

The project is a state-of-the-art advancement of multi biometrics, offering an original viewpoint on appearances fusion. In greater detail, a frequency-based method results in a identical biometric vector incorporate sclera vein and fingerprint vein data. Consecutively a hamming-distancebased matching algorithm deals with the combined homogenous biometric vector. The proposed multimodal scheme achieves interesting results with several commonly used databases.

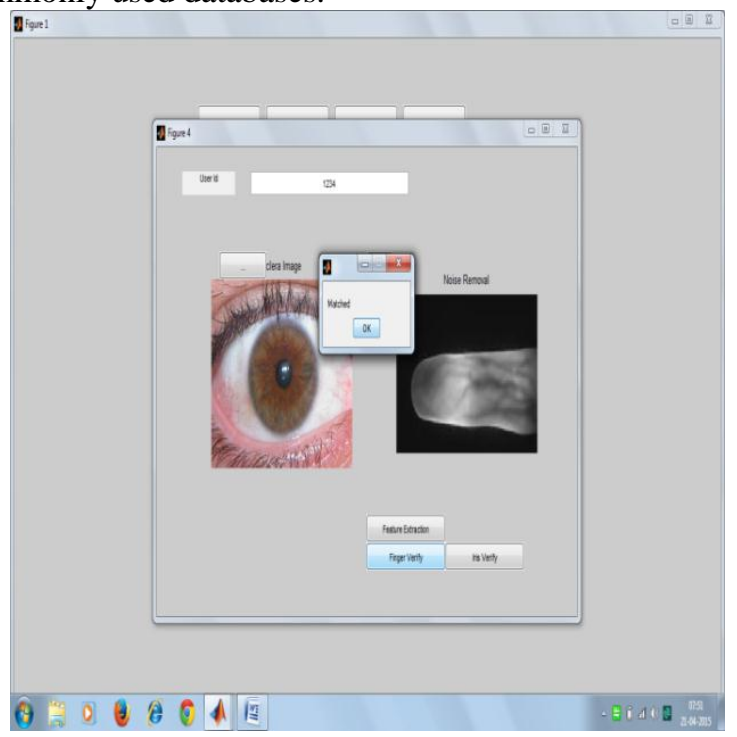

Fig.2 .Finger matching process 


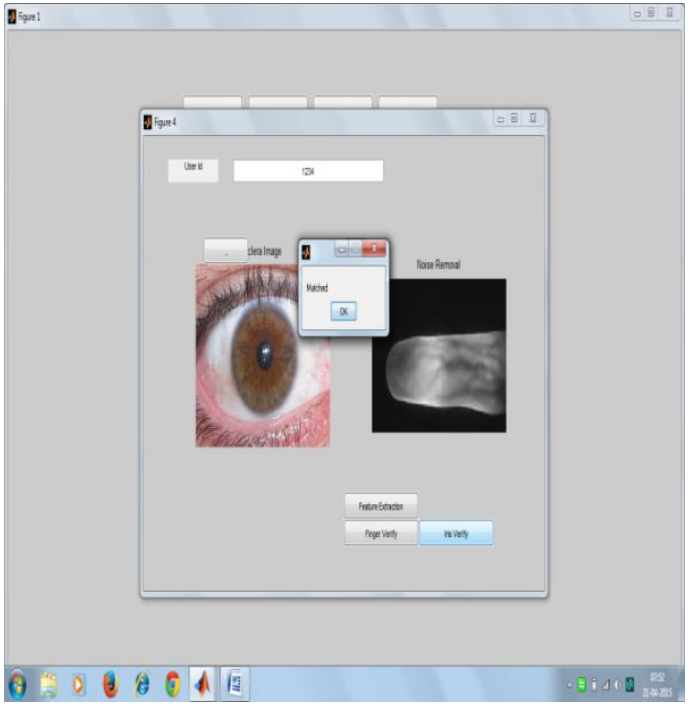

Fig.3.Sclera matching process

The Fig. 2 shows the finger coordinating trial .To obtain the trim for the directory evidence, an individual inserts a finger into an attester terminal containing a near-infrared LED (light- emitting diode) light and a monochrome CCD (charge-coupled device) camera. The hemoglobin in the blood imbibes around-infrared LED light, which makes the strain scheme reaches as a dark decoration of contours. The camera records the representation and the raw data is cyberrnated, authorized and sent to a directory of certified representations. For corroboration purposes, the finger is scanned as before and the data is sent to the database of registered representations for segregation [21] [22]. The corroboration process takes less than two seconds. The Fig .3 shows the Sclera identical process. Blood barge patterns are unique to each individual, as are other biometric dope such as the patterns of the iris [23]. Unlike some biometric schemes, blood barge templates are almost impossible to counterfeit because they are located beneath the sheath's exterior. Biometric schemes based on fingerprints can be fooled with a dummy finger fitted with a copied fingerprint; voice and facial characteristic-based systems can be fooled by recordings and high-resolution representations. The finger strain ID system is much harder to fool because it can only authenticate the finger of a living person.

\section{CONCLUSION AND FUTURE WORK}

Biometric recognition can be used in verification astute, where the biometric scheme classifies a person from the entire enrolled population by searching a database for a match based solely on the biometric. Periodically verification is called "one-to-many" coordinating. Multimodal has several merits over unimodal. Linking the upshots accessed by disparate biometric traits by an effective fusion scheme can significantly improve the overall accuracy of the biometric scheme. Multimodal scheme increases the number of individuals that can concede. It provides hindrance versus kidding. The suggested work includes Sclera and Fingerprint [19][20]as their Multimodal biometric traits for continuous certification of the user.. The proposed method dramatically improves the matching efficiency without compromising recognition accuracy. The sclera segmentation algorithm could be refined. The registration algorithm could be improved by incorporating a 3-D model. For getting better result, Gabor wavelets can be used, also by using different identical methods the recognition rate can be checked.

\section{REFERENCES}

[1]. Yong Lin, Yingzi Du, Senior Member, IEEE, Zhi Zhou, Student Member, IEEE, and N.Luke Thomas:"An Efficient Parallel Approach for Sclera Vein Recognition", IEEE Transactions on Information Forensics, and Security, Vol. 9, No. 2, Feb 2014.

[2]. L. Hong, A. K. Jain, "Integrating faces and fingerprints for personal identification," IEEE Trans. Pattern Analysis Machine Intel., Vol. 20, pp. 1295-1307, December 1998.

[3]. Jain, A., Hong, L., \& Pankanti, S. (2000). "Biometric Identification". Communications of ACM, 43(2), p. 91-98. DOI $10.1145 / 328236.328110$

[4]. K. Jain, R. Bolle, and S. Pankanti (Eds) 1999, "BIOMETRICS: Personal Identification in Networked Society," Kluwer Academic Publishers

[5]. A. K. Jain, A. Ross, and S. Prabhakar, 2004, "An Introduction to Biometric Recognition", IEEE Transactions on Circuits and Systems for Video Technology, Special Issue on Image And VideoBased Biometrics, vol. 14, No. 1, pp. 4-20

[6]. Weaver, A.C. (2006). "Biometric Authentication", Computer, 39 (2), p. 96-97. DOI 10.1109/MC.2006.47

[7]. Jain, L.C. et al. (Eds.). 1999. Intelligent Biometric Techniques in Fingerprint and Face Recognition. Boca Raton, FL: CRC Press.

[8]. Nalini Ratha and Ruud Bolle, Automatic Fingerprint Recognition Systems (Springer: New York, 2004).

[9]. C. W. Oyster, The human eye: structure and function. Sunderland, MA: Sinauer Associates, 1999.

[10]. R. Derakhshani, A. Ross, and S. Crihalmeanu, "A New Biometric Modality Based on Conjunctival Vasculature," Proc. of Artificial Neural Networks in Engineering, 2006.

[11]. M.-K. Hu, "Visual pattern recognition by moment invariants," IRE Transactions on Information Theory, vol. 8, pp. 179-187, 1962.

[12]. R. Derakhshani and A. Ross, "A Texture-Based Neural Network Classifier for Biometric Identification using Ocular Surface Vasculature," in Proc. of the International Joint Conference on Neural Networks, Orlando, FL, 2007, pp. 2982- 2987.

[13]. S. Crihalmeanu, A. Ross, and R. Derakhshani, "Enhancement and Registration Schemes for Matching Conjunctival Vasculature," in Proceedings of the Third International Conference on Advances in Biometrics Alghero, Italy: Springer- Verlag, 2009.

[14]. US Patent no. 7,526,111 "Personal Identification Device and Method"

[15]. David Mulyono, and Horng Shi Jinn, "A Study of Finger Vein Biometric for Personal Identification", Proceedings of the IEEE International Symposium on Biometrics and Security Technologies (ISBAST 2008),pp. 1-8, 2008.

[16]. Wenming Yang, Qing Rao, and Qingmin Liao, "Personal Identification for Single Sample using Finger Vein Location and Direction Coding", Proceedings of the IEEE International Conference on Hand-based Biometrics (ICHB), pp. 1-6, 17-18 March 2011.

[17]. Hua-Bin Wang, and Liang Tao, "Novel Algorithm for Enhancement of Hand Vein Images based on Adaptive Filtering and Retinex Method", Proceedings of the IEEE International Conference on Information Science and Technology (ICIST), Wuhan, Hubei, China, pp.857-860, 23-25 March 2012

[18]. S. Crihalmeanu and A. Ross, "Multispectral scleral patterns for ocular biometric recognition," Pattern Recognit. Lett.vol. 33, no. 14, pp. 1860-1869, Oct. 2012.

[19]. Desong Wang, Jianping Li, and Gokhan Memik, "User Identification based on Finger-vein Patterns for Consumer Electronics Devices", IEEE Transactions on Consumer Electronics, Vol. 56, No. 2, pp. 799-804, May 2010.

[20]. Z. Zhou, E. Y. Du, N. L. Thomas, and E. J. Delp, "A new human identification method: Sclera recognition,” IEEE Trans. Syst., Man, Cybern. A, Syst., Humans, vol. 42, no. 3, pp. 571-583, May 2012. 
[21]. Gongping Yang, Xiaoming Xi, and Yilong Yin, "Finger Vein Recognition Based on a Personalized Best Bit Map", Journal on Sensors, Vol. 12, pp. 1738-1757, doi: 10.3390/s120201738, 2012.

[22]. Naoto Miura, Akio Nagasaka, and Takafumi Miyatake, "Feature Extraction of Finger-vein Patterns based on Repeated Line Tracking and its Application to Personal Identification", Machine Vision and Applications, Vol. 15, pp. 194-203, 2004.

[23]. R. N. Rakvic, B. J. Ulis, R. P. Broussard, R. W. Ives, and N. Steiner,"Parallelizing iris recognition," IEEE Trans. Inf. Forensics Security, vol. 4, no. 4, pp. 812-823, Dec. 2009. 